\title{
Effect of Incoherent Pumping on Phase-dependent Absorption Property of Atomic Systems
}

\author{
Aiyun $\mathrm{Li}^{\mathrm{a}}$ and Fengshou Liu ${ }^{\mathrm{b}}$ * \\ Opto-Electronic Engineering College , Zaozhuang University, 277160,China \\ aliaiyun@126.com, bfegnshouliu@126.com \\ * The corresponding author
}

\begin{abstract}
Keywords: Incoherent pumping; Lasing without inversion; Relative phase; Gain
\end{abstract}
\begin{abstract}
We find that in the open and closed ladder systems with vacuum induced coherence, the relative phase between the probe and driving fields has an important manipulation role on the absorption property, while the incoherent pumping has a considerable effect on the phase-dependent absorption property. In the both systems, by adjusting the incoherent pumping the switching from absorption to gain and from lasing with inversion to lasing without inversion (LWI) can be realized and the largest LWI gain can be gotten. However, when the incoherent pumping is absent, for any value of the relative phase, LWI still can't be obtained in the closed system but can in the open system; moreover, in the open system, for suitable values of the relative phase, LWI gain gotten when the incoherent pumping is absent is obviously larger than that obtained when the incoherent pumping presents.
\end{abstract}

\section{Introduction}

Quantum coherence and interference in atomic systems can lead to many interesting effects such as lasing without inversion (LWI), electromagnetically induced transparency (EIT), coherent population trapping (CPT) and so on, in which LWI gets special attention due to its important application value[1]. A kind of coherence can be created by interference of spontaneous emission (usually called as the vacuum-induced coherence (VIC) or spontaneously generated coherence (SGC)), and it depends on the nonorthogonality of dipole matrix elements. Recently, some people have studied the effects of VIC on EIT, LWI, unexpected population inversion, transient process, optical bistability and multistability etc.[2-7]. It has been shown that atomic systems with VIC are sensitive to the relative phase of the applied fields[8-11]. Niu[12] has shown that the incoherent pumping plays a very important role and gives the necessary condition of the realization of lasing without inversion (LWI) in a closed ladder system without VIC. Qian et al. also have obtained the same conclusion for a closed ladder system with VIC. In this paper we first put forward a scheme of an open ladder system with VIC coreesponding to the closed system, and then compare the role of incoherent pumping on the phase-dependent absorption (gain) property in the both systems. We find that varying value of the incoherent pumping has a remarkable effect on the phase -dependent LWI gain of the probe field in the both system. Our study revealed that even considering the effect of the relative phase, when the incoherent pumping is absent, LWI still can't be obtained in the closed system, however, LWI can be realized in the open system.

\section{Model and Motion Equations}

The open ladder-type three level atomic system with VIC considered here is shown in Fig.1. The transition $|1\rangle \rightarrow|2\rangle$ is coupled by a weak probe field of frequency $\omega_{\mathrm{a}}$ with Rabi frequency $\mathrm{g}=\vec{\mu}_{12} \cdot \vec{\varepsilon}_{a} / \hbar$ while the transition $|2\rangle \rightarrow|3\rangle$ is coupled by a strong driving field of frequency $\omega_{\mathrm{b}}$ with Rabi frequency $G=\vec{\mu}_{23} \cdot \vec{\varepsilon}_{b} / \hbar$. The level $|2\rangle(|3\rangle)$ spontaneously decays to the level $|1\rangle$ $(|2\rangle)$ at the rate $\gamma_{1}\left(\gamma_{2}\right)$. An incoherent pump with a pumping rate $\mathrm{R}$ is applied between levels $|1\rangle$ 
and $|3\rangle$. The atomic injection rates for levels $|1\rangle$ and $|2\rangle$ are $J_{1}$ and $J_{2}$, respectively. The atomic exit rate from the cavity is $r_{0}$. In the following discussion, we always make $J_{1}+J_{2}=r_{0}$ for keeping the total number of the atoms as a contant. In such a case, the density-matrix motion equations in a rotating frame can be written as

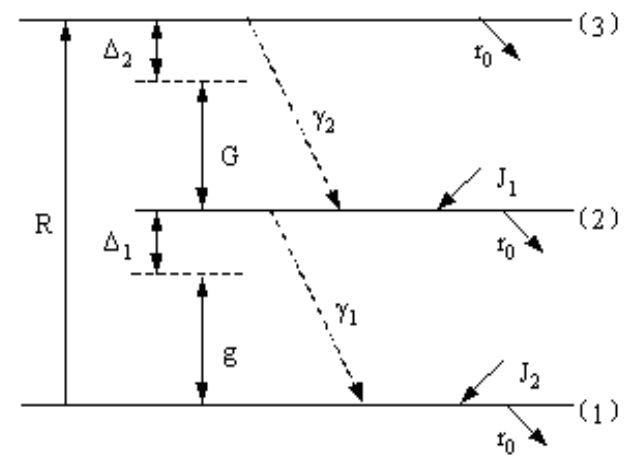

Figure.1. The schematic diagram of an open ladder-type three-level atomic system with VIC.

$$
\begin{aligned}
& \dot{\rho_{11}}=-\left(2 R+r_{0}\right) \rho_{11}+2 \gamma_{1} \rho_{22}+i g^{*} \rho_{21}-i g \rho_{12}+J_{1} \\
& \left.\dot{\rho_{22}}=2 \gamma_{2} \rho_{33}-\left(2 \gamma_{1}+r_{0}\right) \rho_{22}-i g^{*} \rho_{21}+i g \rho_{12}-i G \rho_{23}+i G^{*} \rho_{32}\right)+J_{2} \\
& \dot{\rho_{33}}=2 R \rho_{11}-\left(2 \gamma_{2}+r_{0}\right) \rho_{33}+i G \rho_{23}-i G^{*} \rho_{32} \\
& \dot{\rho_{23}}=-\left(\gamma_{1}+\gamma_{2}+i \Delta_{2}\right) \rho_{23}+i G^{*}\left(\rho_{33}-\rho_{22}\right)+i g \rho_{13} \\
& \dot{\rho_{12}}=-\left(R+\gamma_{1}+i \Delta_{1}\right) \rho_{12}+i g^{*}\left(\rho_{22}-\rho_{11}\right)-i G \rho_{13}+2 p \sqrt{\gamma_{1} \gamma_{2}} \eta \rho_{23} \\
& \dot{\rho_{13}}=-\left[\gamma_{2}+R+i\left(\Delta_{1}+\Delta_{2}\right)\right] \rho_{13}-i G^{*} \rho_{12}+i g^{*} \rho_{23}
\end{aligned}
$$

constrained by $\rho_{11}+\rho_{22}+\rho_{33}=1$ and $\rho^{*}{ }_{m n}=\rho_{n m}$. Where, $\rho_{i i}$ is the atomic population of state $|i\rangle$, and $\rho_{i j}$ is the atomic polarization between states $|i\rangle$ and $|j\rangle ; \Delta_{1}$ and $\Delta_{2}$ denote the frequency detuning of the probe and the driving fields, respectively. The inclusion of two coupling fields of different frequencies would lead to the optical Bloch equation with additional term $2 p \sqrt{\gamma_{1} \gamma_{2}} \eta \rho_{23}$, which presents the effect of VIC. Where $p=\cos \theta, \theta$ is the angle between the two induced dipole moments $\vec{\mu}_{12}$ and $\vec{\mu}_{23}$. Using the restriction that each of the linearly polarrized field should only couple one of the optical transitions, we can find the Rabi frequencies are connected to the parameter $p$ by the ralation $G=G_{0} \sqrt{1-p^{2}}=G_{0} \sin \theta$ and $g=g_{0} \sqrt{1-p^{2}}=g_{0} \sin \theta$, with $G_{0}=\left|\vec{\mu}_{23}\right|\left|\vec{\varepsilon}_{b}\right| / \hbar$ and $g_{0}=\left|\vec{\mu}_{12}\right|\left|\vec{\varepsilon}_{a}\right| / \hbar$. It is obviously that when $\eta=1$, the effect of VIC presents, the strength of VIC will vary with $\theta$. When $J_{1}=J_{2}=r_{0}=0$, Eqs.(1) is the density-matrix motion equations discribing the corresponding closed three-level ladder system with VIC. Otherwise $\eta=0$, the VIC effect is absent, Eqs.(1) becomes an density-matrix motion equations discribing an open three-level ladder system without VIC ${ }^{[20]}$. Due to VIC, the absorption (gain) property of the system denpents not only on the amplitudes and detunings but also phases of the the probe and driving fields, thus we have to treat Rabi frequencies as complex parameters. Let $\phi_{p}$ and $\phi_{c}$ denote the phases of the probe and driving fields, respectively, then we have $g=g_{p} \exp \left(i \phi_{p}\right)$ and $G=G_{c} \exp \left(i \phi_{c}\right) \quad\left(g_{p}\right.$ and $G_{c}$ are real parameters) and the relative phase between the probe and the driving fields is $\Phi=\phi_{p}-\phi_{c}$. Let $\tilde{\rho}_{i i}=\rho_{i i}$, 
$\tilde{\rho}_{12}=\rho_{12} \exp \left(i \phi_{p}\right), \phi=\phi_{c}+\phi_{p}$ and $\tilde{\rho}_{23}=\rho_{23} \exp \left(i \phi_{c}\right)$, we get the motion equations for the redefined density matrix elements $\tilde{\rho}_{i j}$, which are found to be identical to Eqs. (1) except that $\eta, g$ and $G$ are replaced by $\eta_{\Phi}=\eta e^{i \Phi}, g_{p}$ and $G_{c}$, respectively. The steady-state solutions for $\tilde{\rho}_{i j}$ can be found by setting the time derivatives to zero and reducing the Eqs. for $\tilde{\rho}_{i j}$ to a set of coupled $9 \times 9$ algebraic equations after splitting into real and imaginary parts. These equations can be treated in all orders using the symbolic computation package Mathemetica or Maple. The absorption (gain) of the medium correspond to imaginary parts of $\tilde{\rho}_{12}$. If $\operatorname{Im}\left(\tilde{\rho}_{12}\right)>0$, the system exhibits gain for the probe field; if $\operatorname{Im}\left(\tilde{\rho}_{12}\right)<0$, the probe field is attenuated. When $\operatorname{Im}\left(\tilde{\rho}_{12}\right)>0$ and $\tilde{\rho}_{22}-\tilde{\rho}_{11}<0$, LWI can be realized; if $\operatorname{Im}\left(\tilde{\rho}_{12}\right)>0$ and $\tilde{\rho}_{22}-\tilde{\rho}_{11}>0$, the lasing with inversion occurs.

\section{Numerical Results and Discussion}
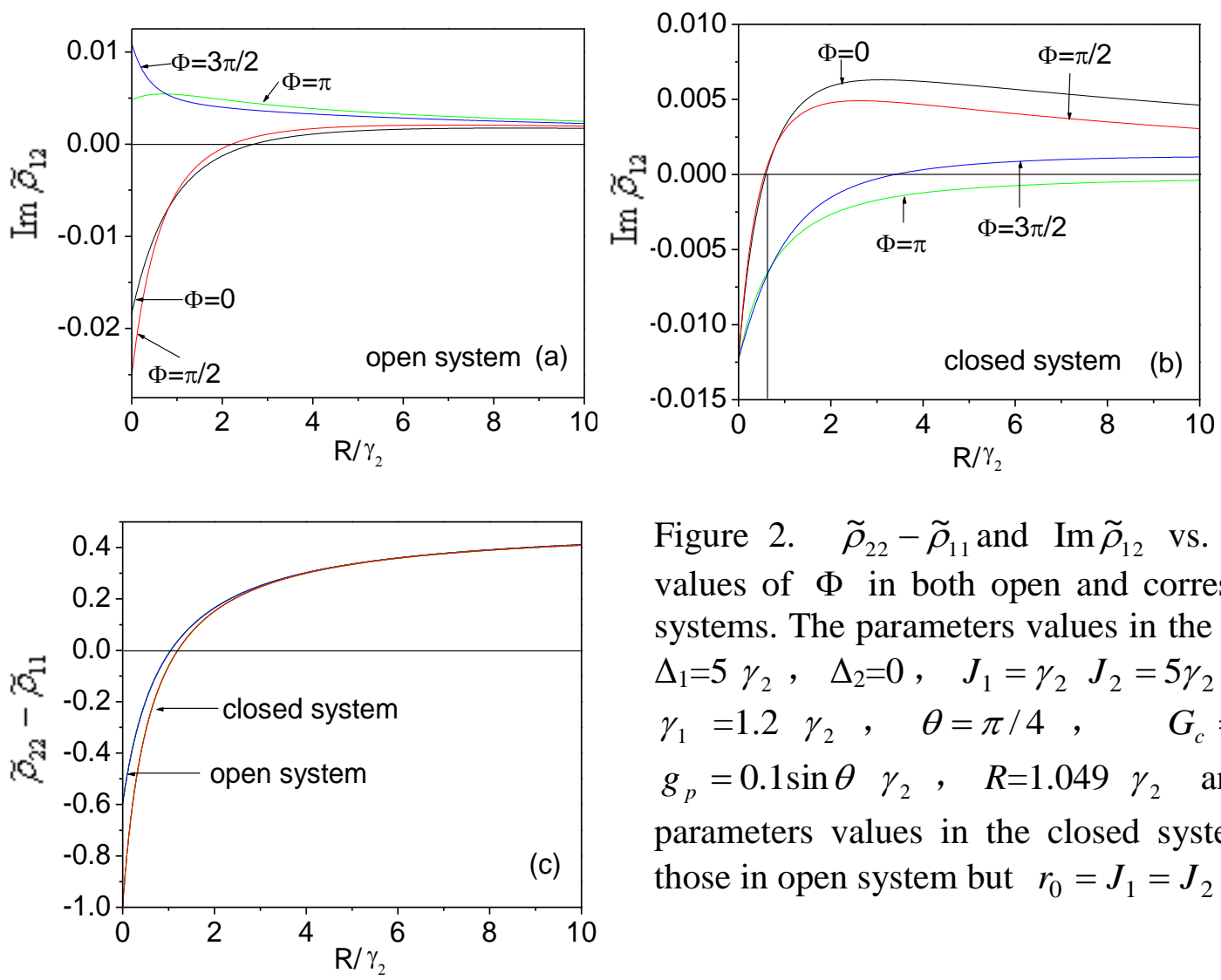

Figure 2. $\tilde{\rho}_{22}-\tilde{\rho}_{11}$ and $\operatorname{Im} \tilde{\rho}_{12}$ vs. R for different values of $\Phi$ in both open and corresponding closed systems. The parameters values in the open system are $\Delta_{1}=5 \gamma_{2}, \Delta_{2}=0, \quad J_{1}=\gamma_{2} J_{2}=5 \gamma_{2}, \quad r_{0}=0.6 \gamma_{2}$, $\gamma_{1}=1.2 \gamma_{2}, \theta=\pi / 4, \quad G_{c}=5 \sin \theta \quad \gamma_{2}$, $g_{p}=0.1 \sin \theta \quad \gamma_{2}, R=1.049 \quad \gamma_{2}$ and $\eta=1 ;$ the parameters values in the closed system are same as those in open system but $r_{0}=J_{1}=J_{2}=0$.

Fig.2 illustrates $\tilde{\rho}_{22}-\tilde{\rho}_{11}$ and $\operatorname{Im} \tilde{\rho}_{12}$ versus the incoherent pumping rate $\mathrm{R}$ for different values of $\Phi$ in both open and corresponding closed systems. Fig. 2 shows that in the open and closed systems the relative phase has an important manipulation role on the absorption property, while the incoherent pumping has a consideable effect on the phase-dependent absorption property. From Fig.2 (a) and (c) we can see that: in the open system, corresponding to $\Phi=0$ and $\Phi=\pi / 2$, with value of $\mathrm{R}$ increasing, the probe field exhibits successively absorption without inversion, absorption with inversion, lasing with inversion; however, corresponding to $\Phi=\pi$ and $\Phi=3 \pi / 2$ the probe gain always can be gotten for any value of $\mathrm{R}$, while the conversion from LWI gain to gain with inversion occurs when value of $\tilde{\rho}_{22}-\tilde{\rho}_{11}$ change from negative to positive with value of $\mathrm{R}$ increasing. Figs.2 (b) and (c) show that in the corresponding closed system, when value of $\mathrm{R}$ is small, the probe field exhibits only absorption for any value of $\Phi$; with value of R increasing, the gain without- and with- inversion can successively be realized for $\Phi=0$ and $\Phi=\pi / 2$; but for the case of $\Phi=3 \pi / 2$, only the gain with inversion occurs, for the case of $\Phi=\pi$, only the probe 
absorption appears. From Fig.2 we can also see that in the open system we can obtain larger LWI gain than that obtained in the corresponding closed system. Fig. 2 tell us that that even considering the effect of the relative phase between the probe and driving fields, the incoherent pumping is still the necessary condition of realizing LWI in a closed ladder system with VIC; but for the corresponding open system, when the incoherent pumping is absent, LWI also can be obtained for $\Phi=\pi$ and $\Phi=3 \pi / 2$; moreover, for the case $\Phi=3 \pi / 2$, LWI gain gotten when the incoherent pumping is absent is obviously larger than that obtained when the incoherent pumping presents. From Fig.2 (c) we know that, influence of the incoherent pumping on the populations in the open and closed system just has a saller differene.

\section{Conclusions}

In conclusions, in this paper, we investigate mainly the effect of incoherent pumping on the phase-dependent absorption property in the open and closed ladder systems with vacuum induced coherence by using numerical calculation results from the analytical representatives of $\operatorname{Im} \tilde{\rho}_{12}$ and $\tilde{\rho}_{22}-\tilde{\rho}_{11}$. We find that, the incoherent pumping play a very important role to get inversionless gain of the probe field and produce LWI in the both systems; but the influence of the incoherent pumping in the both systems has considerable difference, specially, when the incoherent pumping is absent, even considering the effect of the relative phase between the probe and driving fields, LWI still can't be obtained in the closed system but can in the open system; moreover, in the open system, for some values of the relative phase, LWI gain when the incoherent pumping is absent is obviously larger than that obtained when the incoherent pumping present.

\section{Acknowledgements}

This work was supported by the Program of independent innovation and achievement transformation plan for Zaozhuang (No. 2016GH19), Science and Technology Program of zaozhuang ((No. 2016GX31).

\section{References}

[1] J. Mompart and R. Corbalan: Optics B, Vol. 2 (2000) No.2, p.27.

[2] S.Q. Gong, E. Paspalakis and P.L. Knight: J. Mod. Opt, Vol. 45 (1998) No.3, p.2433.

[3] E. Paspalakis and P.L. Knight: Phys. Rev. A, Vol. 61 (2000) No.4, p.045802.

[4] Peng L. Individual Vision and Peak Distribution in Collective Actions [J]. Communications in Nonlinear Science and Numerical Simulation. 2017, 47: 238-252.

[5] W.H. Xu, J.H. Wu and J.Y. Gao: Phys.Rev. A, Vol. 66 (2002) No.6, p.063812.

[6] Y. Wu, L. Wen and Y. Zhu: Opt. Lett, Vol. 28 (2003) No.8, p.631.

[7] H.M. Ma, S.Q. Gong and C.P. Liu: Opt. Commun, Vol. 223 (2003) No.2, p.97.

[8] C.P. Liu, S.Q. Gong and X.J. Fan: Opt. Commun, Vol. 231 (2004) No.2, p.289.

[9] D.C. Cheng, C.P. Liu and S.Q. Gong: Phys.Lett. A, Vol. 322 (2004) No.3, p.244.

[10] Y. Wu, M.G. Payne and E.W. Hagley: Opt. Lett, Vol. 29 (2004) No.19, p.2294.

[11]J. Qian, C.P. Liu and S.Q. Gong: Chin. Opt. Lett, Vol. 3 (2005) No.3, p.308.

[12]M.A. Anton, O.G. Calderon and F. Carreno: Phys Rev. A, Vol. 72 (2005) No.2, p.23809.

[13]Y.P. Niu and S.Q. Gong: Phys. Rev. A, Vol. 73 (2006) No.5, p.53811. 